\title{
CAESAREAN SECTION RATE AND FREQUENCIES OF INDICATIONS USING ROBSON-TEN CLASSIFICATIONS
}

\author{
Sadia Ali $^{1}$, Shazia Khattak ${ }^{2}$, Rabeea Sadaf ${ }^{3}$, Shamshad Begum ${ }^{4}$, Nasreen Kishwar $^{5}$
}

\section{ABSTRACT:}

\section{OBJECTIVES:}

To determine the caesarean section rate (CSR) and frequency of different indications of caesarean section (CS) in a tertiary care hospital.

\section{METHODOLOGY:}

A retrospective study done in the Department of Obstetrics and Gynecology Hayatabad Medical Complex Hospital Peshawar, a tertiary care hospital, from a period of $1^{\text {st }}$ January 2019 till $31^{\text {st }}$ December 2019. The required data was collected from the patient's hospital records (clinical charts) with the consent of the hospital ethical committee.

\section{RESULTS:}

The total number of deliveries over the study period was 5611. Out of these 1258 patients were delivered through caesarean section (CS), giving a CSR of $22 \%$. The main contributing groups in our study were Robson Groups R5 (multiparous with prior CS, singleton, cephalic and >37 weeks), R1 (nulliparous, cephalic, singleton $>37$ weeks in spontaneous labor or CS) and R6 (all nulliparous breeches) with percentages of $21.1 \%, 17.5 \%$ and $12.9 \%$ respectively.

\section{CONCLUSION:}

Our study showed Robson Groups 5, 2 and 6 as the major contributors, focusing on these groups could have an impact on decreasing the cesarean section rate in future. Limiting the primary cesarean section rate can affect the overall cesarean section rate (CSR).

KEYWORDS: Caesarean Section, Robson, Maternal Morbidity, Obstetrics, Gynecology

How to cite this article:

Ali S, Khattak S, Sadaf R, Begum S, Kishwar N. Caesarean Section Rate and Frequencies of Indications Using Robson -Ten

Classifications. J Gandhara Med Dent Sci. 2021;8(4): 43-47

https://doi.org/10.37762/jgmds.8-4.259

\section{Correspondence:}

${ }^{2}$ Shazia Khattak, Assistant Professor, Department of Gynecology and Obstetrics, Hayatabad Medical

Complex, Peshawar

Contact: 0336-3353592

Email: shaziakhattak85@gmail.com

${ }^{1}$ Assistant Professor, Department of Gynecology and

Obstetrics, Hayatabad Medical Complex, Peshawar

${ }^{3}$ Associate Professor, Department of Gynecology and

Obstetrics, Hayatabad Medical Complex, Peshawar

${ }^{4}$ Professor, Department of Gynecology and Obstetrics,

Hayatabad Medical Complex, Peshawar

${ }^{5}$ Assistant Professor, Department of Gynecology and

Obstetrics, Hayatabad Medical Complex, Peshawar

\section{INTRODUCTION:}

In the field of obstetrics, caesarean section (CS) has emerged as a lifesaving surgical procedure both for the mother and the baby ${ }^{1}$, appropriately employed it has shown to effectively reduce the maternal and infant mortality ${ }^{2}$. However, like any surgical procedure CS is not without risks both for the baby and mother, neonates born through CS have shown increased risks of respiratory distress syndrome (RDS) and increased admission to $\mathrm{NICU}^{3}$. Maternal complications 
include anesthetic complications, thromboembolism, wound infection, long term effects on her future pregnancy e.g. risk of scar dehiscence, uterine rupture, and placental abnormalities like placenta accrete leading to life threatening emergencies. In short CS has a lifelong impact on the reproductive life of a female ${ }^{4,5}$. Caesarean section rates (CSR) are on a rise globally. A gradual upward shift in the caesarean rates have been observed over the past five decades, where it has increased from $5 \%$ in 1940 to $15 \%$ in 1970 and 1980 's ${ }^{6}$. The Lancet series (2018) highlighted this issue by giving us the figures of year 2000 where CSR was $12.1 \%$ and it almost doubled in 2015 with a CSR of $21.1 \%$. According to this report, the CSR was highest in Latin America and Caribbean (44.3\%) and lowest in West and Central Africa $(4.1 \%)^{7}$. Almost similar patterns in the CSR are observed in Pakistan, where the CSR has increased from $2.7 \%$ in 1990 and 1991 to $15.8 \%$ in $2012-2013^{8}$. WHO has recommended keeping the CSR at 10 to $15 \%$, as rates exceeding this figure have not proven any beneficial effects for the mother and baby and no reduction in the rates of perinatal or maternal mortality were observed in areas with caesarean section above $10 \%{ }^{9}$. Wide variations in CSR between different regions and among the health facilities suggest lack of protocols and a variation in practices ${ }^{10}$. For better understanding of the rates of caesarean section in different regions and health facilities we need an internationally accepted classification system for CS. WHO comes to the rescue by recommending Robson classification system, also known as Robson 10 , as a global standard for assessing, monitoring, and comparing cesarean section rates between healthcare facilities ${ }^{9}$. A systematic review conducted by WHO in 2011 concluded that Robson classification is the most appropriate system to fulfill the current local and international needs ${ }^{9}$. Robson 10 classifies patients in ten groups using their obstetrical characteristics, which allows for comparison of the CSR between different health facilities with better understanding of the indications and less confounding factors ${ }^{10}$. The purpose of this study is to determine the CSR using Robson 10 classification for identification of the areas of focus. Using this information as a tool we can effectively reduce the CSR.

\section{METHODOLOGY:}

This is a retrospective study carried out in the Department of Gynecology and Obstetrics Hayatabad Medical Complex, a tertiary care hospital, of Peshawar city over a period of one year from $1^{\text {st }}$ January 2019 to $31^{\text {st }}$ December 2019. Approval from the hospital ethical committee was taken before proceeding for the research. The required data was obtained from the patient's hospital records (clinical charts). All the collected data was classified according to Robson's classification system, ${ }^{11}$ analyzed using simple statistics and results calculated in terms of percentages. All the patients delivering through cesarean section during the study period were included. Relevant parameters like gestational age, parity, previous scar, fetal presentation (breech, cephalic, transverse) and number of fetuses (singleton/multiple) were noted. Exclusion criteria: patients delivering normally or via forceps or vacuum and those delivering preterm were excluded from the study.

\section{RESULTS:}

The total number of deliveries over the study period were 5611. Out of these 1258 patients were delivered through caesarean section, giving a rate of $22 \%$. According to our study, 265 patients delivered by CS were from R5 (multiparous, singleton with $>37$ weeks and previous scar), giving a CSR of $21.1 \% .220$ patients were from R1 (nulliparous with singleton, cephalic pregnancy and gestation of $>37$ weeks, in spontaneous labor), giving a CSR of $17.5 \%$. The other dominant group was the nulliparous breeches (R6) with 162 $(12.9 \%)$ patients delivered by CS. 
Table 1: CSR and Frequencies Indicated through Robson-Ten Classification

\begin{tabular}{|c|c|c|}
\hline $\begin{array}{c}\text { Groups } \\
\mathbf{R} \text { ( }\end{array}$ & Clinical Characteristics & Results (\%) \\
\hline $\mathbf{1}$ & Nulliparous, Singleton, Cephalic, >37 Weeks, Spontaneous Labor & $17.5 \%$ \\
\hline $\mathbf{2}$ & Nulliparous, Singleton, Cephalic, >37 Weeks Induced Labor or Cesarean Section Before Labor & $10.3 \%$ \\
\hline $\mathbf{3}$ & Multiparous Without Previous Cesarean Section, Singleton, Cephalic, >37 Weeks, Spontaneous \\
Labor & $9.9 \%$ \\
\hline $\mathbf{4}$ & Multiparous Without Previous Cesarean Section, Singleton, Cephalic, >37 weeks, Induced Labor or & $6.0 \%$ \\
\hline $\mathbf{5}$ & Cesarean Section Before Labor & $21.1 \%$ \\
\hline $\mathbf{6}$ & Multiparous With Prior Cesarean Section, Singleton, Cephalic, >37 weeks & $12.9 \%$ \\
\hline $\mathbf{7}$ & All Multiparous Breeches (Including Previous Cesarean Section) & $7.1 \%$ \\
\hline $\mathbf{8}$ & All Multiple Pregnancies (Including Previous Cesarean Section) & $5.1 \%$ \\
\hline $\mathbf{9}$ & All Pregnancies With Abnormal Lie (Transverse or Oblique Lie) (Including That Previous Cesarean \\
& Section) & $5.9 \%$ \\
\hline $\mathbf{1 0}$ & All Single, Cephalic, <37 Weeks, Including Previous CS & $3.9 \%$ \\
\hline
\end{tabular}

\section{DISCUSSION:}

Rising CSR has become a global health problem. Wide variations have been observed in the rates of CS across the globe. To overcome this discrepancy WHO recommended the use of Robson 10 classification system as a uniform and standardized system for the analysis and audit of cesarean section. CSR of this study is $22 \%$, higher than the WHO recommended rate of 10 to $15 \%$, but rates even higher than ours were observed in certain other hospitals of Pakistan e.g., $33 \%$ in PIMS $(2016-2017)^{12}, 49 \%$ in Fauji Foundation Pindi, ${ }^{13} 31.26 \%$ in Agha Khan Hospital Karachi $(2014)^{14}, 46.7 \%$ in $\mathrm{CMH}$ Abbottabad $(2018)^{15}$. A study done at Kahuta research laboratories has shown an unacceptably high rate of $64 \%$ for the year 2017-2018 ${ }^{15}$. Majority of the studies have reported Robson groups (R) 1, 2 and 5 as the main contributors ${ }^{16,17}$. A study done in $\mathrm{LRH}$ Peshawar has reported a rate of $21.7 \%$ (2010$2011),{ }^{17}$ this is somewhat closer to the $22 \%$ rate of our current study. Our study showed somewhat similar results with groups 1, 5 and 6 as the leading groups. Robson (R5) was the major contributor in our study with $21.5 \%$; some studies have reported even higher rates for Group 5 such as $80.3 \%, 58.2 \%, 61 \%$ and $36 \%{ }^{16,18}$. The different trends seen in the rates may be because of different labor ward protocols but an overall increase rate seen in the R5 group shows that there is generally a fear of trial of labor in a previous scarred uterus and patients opting for elective CS due to the risk of rupture ${ }^{19}$. Promoting VBAC (vaginal birth after CS) can effectively lead to lowering of CS rate in R5 group, studies have shown that VBAC (vaginal birth after CS) is safe when conducted in a controlled environment of hospital ${ }^{20}$. A study from a tertiary care hospital showed a reduction of CS rate from $63 \%$ to $57 \%$ with successful VBAC (vaginal birth after $\mathrm{CS})^{21}$. This shows that offering trials of labor to the patients with non-recurrent indications can effectively reduce the cesarean section rate. Groups R1 and R2 comprise of nulliparous patients. Our study gave R1 (nulliparous, single, cephalic, $>37$ weeks in spontaneous labor) as the second major contributing group with $17.5 \%$ rate of CS, R2 (nulliparous, single, cephalic, >37 weeks, induced or cesarean section before labor) was showing a rate of $10.3 \%$ in our study but in certain other studies it was the second leading group after group 
$5^{17,18,21}$. Lowering rate of primary CS, i-e R1 and R2 can positively affect the overall CS rate $^{22}$. Implementing strict labor protocols, use of partograph for labor monitoring ${ }^{23}$, judicious use and correct interpretation of CTG, ${ }^{24}$ can affect the rate of CS in this group. R6 and R7 comprise of nulliparous and primiparous breeches. Our study showed R6 to be the third leading group with $12.9 \%$. The results of the term breech trial led to a rise in caesarean deliveries done for breech fetuses. External cephalic version has shown a positive role in decreasing CS rate ${ }^{25}$. Use of ECV and improving skills in breech vaginal delivery can control the rate in these groups. R8 comprises multiple pregnancy, the decision about the mode of delivery depends upon the presentation of the first baby in twin pregnancy. In cases of first breech, in twins, and in higher order pregnancies CS is the best management. Nothing much can be done in this group and R9 where the fetus presents with an abnormal lie and CS is the recommended management. The contribution by these groups to the overall CS rate is small owing to their small size.

\section{CONCLUSION:}

Using Robson as a standardized classification system, target areas can be identified where improvement can be made with implementation of properly designed protocols, with a hope to achieve the WHO recommended rate of CS. Our study showed R5, R2 and R6 as the major contributors.

\section{LIMITATIONS:}

It's a small-scale study, done in one hospital only, for better understanding larger scale studies including more than one hospital are needed.

\section{CONFLICT OF INTEREST: None}

FUNDING SOURCES: None

\section{REFRENCES:}

1. Gonda A, Bukhari S, Karim MT, Karim $\mathrm{S}$. Frequency of caesarean section at a tertiary care hospital. J Sheikh Zayed Med Coll. 2017;8(3):1248-50.

2. Tahir N, Adil M, Fatima S, Khan S. Caesarian sections: frequency and indications at peripheral tertiary care hospital. Pak Armed Forces Med J. 2018;68(2):273-9.

3. Khasawneh W, Obeidat N, Yusef D, Alsulaiman JW. The impact of cesarean section on neonatal outcomes at a university-based tertiary hospital in Jordan. BMC Pregnancy Childbirth. 2020;20:1-9.

4. Mascarello KC, Horta BL, Silveira MF. Maternal complications and cesarean section without indication: systematic review and meta-analysis. Rev Saude Publica. 2017;51:105.

5. Chen S, Cheng W, Chen Y, Liu X. The risk of abnormal placentation and hemorrhage in subsequent pregnancy following primary elective cesarean delivery. J Matern Fetal Neonat Med. 2020;33(21):3608-13.

6. Kant A, Mendiratta S. Classification of cesarean section through Robson criteria: an emerging concept to audit the increasing cesarean section rate. Int J Reprod Contracept Obstet Gynaecol. 2018;7(11):4674-7.

7. Boerma T, Ronsmans C, Melesse DY, Barros AJ, Barros FC, Juan L, et al. Global epidemiology of use of and disparities in caesarean sections. Lancet. 2018;392(10155):1341-8.

8. Mumtaz S, Bahk J, Khang YH. Rising trends and inequalities in cesarean section rates in Pakistan: evidence from Pakistan demographic and health surveys, 1990-2013. PLoS ONE. 2017;12(10):e0186563.

9. World Health Organization. World Health Statistics 2015: WHO statement on caesarean section rates. World Health Organization; 2015. $161 \mathrm{p}$.

10. Betrán AP, Ye J, Moller AB, Zhang J, Gülmezoglu AM, Torloni MR. The increasing trend in caesarean section rates: global, regional and national estimates: 1990-2014. PLoS ONE. 2016;11(2):e0148343.

11. Betran AP, Vindevoghel N, Souza JP, Gülmezoglu AM, Torloni MR. A systematic review of the Robson classification for caesarean section: what works, doesn't work and how to improve it. PLoS ONE. 2014;9(6):e97769.

12. Gilani S, Mazhar SB, Zafar M, Mazhar 
T. The modified Robson criteria for caesarean section audit at mother and child health center Pakistan Institute of Medical Sciences Islamabad. J Pak Med Assoc. 2020;70(2):299-303.

13. Imran F, Alia N, Zaman Z. An audit of cesarean section in Fauji Foundation Hospital Rawalpindi. J Soc Obstet Gynaecol Pak. 2016;6:182-6.

14. Bano R, Mushtaq A, Adhi M, Saleem MD, Saif $A$, Siddiqui $A$, et al. Rates of caesarean section and trials and success of vaginal birth after caesarean sections in secondary care hospital. J Pak Med Assoc. 2015;65(1):81-3.

15. Khan MA, Sohail I, Habib M. Auditing the cesarean section rate by Robson's ten group classification system at tertiary care hospital. Prof Med J. 2020;27(04):700-6.

16. Naeem M, Khan MZ, Abbas SH, Khan A, Adil M, Khan MU. Rate and indications of elective and emergency caesarean section; a study in a tertiary care hospital of Peshawar. J Ayub Med Coll Abbottabad. 2015;27(1):151-4.

17. Robson M, Murphy M, Byrne F. Quality assurance: the 10-Group Classification System (Robson classification), induction of labor, and cesarean delivery. Intel J Gynecol Obstet. 2015;131:S23-7.

18. Schemann K, Patterson JA, Nippita TA, Ford JB, Roberts CL. Variation in hospital caesarean section rates for women with at least one previous caesarean section: a population based cohort study. BMC Pregnancy Childbirth. 2015;15(1):179.
19. Senturk MB, Cakmak $Y$, Atac H, Budak MS. Factors associated with successful vaginal birth after cesarean section and outcomes in rural area of Anatolia. Int J Women's Health. 2015;7:693.

20. Imtiaz R, Husain S, Izhar R. Adoption of Robson's Ten Group Classification System (RTGCS) to analyse caesarean section rates at a tertiary care centre in Pakistan. Ann Abbasi Shaheed Hosp Karachi Med Dent Coll. 2018;23(1):46-52.

21. Yadav RG, Maitra N. Examining cesarean delivery rates using the Robson's ten-group classification. J Obstet Gynecol India. 2016;66(1):1-6.

22. Samba A, Mumuni K. A review of caesarean sections using the ten-group classification system (Robson classification) in the Korle-Bu Teaching Hospital (KBTH), Accra, Ghana. Gynecol Obstet (Sunnyvale). 2016;6(385):2161-932.

23. Bo O, Oh O, Osanyin G, Oe O, Ba O. Effect of training on the knowledge and use of the partograph for low risk pregnancies among health workers in a tertiary hospital in Lagos State, Nigeria. $J$ Community Med Primary Health Care. 2018;30(2):47-54.

24. East CE, Begg L, Colditz PB, Lau R. Fetal pulse oximetry for fetal assessment in labour. Cochrane Database Syst Rev. 2014;(10).

25. Rauf B, Ali S. Effectiveness of nifedipine in external cephalic version. J Postgrad Med Inst. 2017;31(4):37882.

\section{CONTRIBUTORS}

1. Sadia Ali - Concept \& Design; Data Acquisition; Data Analysis/Interpretation; Drafting Manuscript; Critical Revision; Supervision; Final Approval

2. Shazia Khattak - Drafting Manuscript; Critical Revision; Supervision

3. Rabeea Sadaf - Data Analysis/Interpretation; Critical Revision; Supervision

4. Shamshad Begum - Data Acquisition; Supervision

5. Nasreen Kishwar - Data Acquisition; Data Analysis/Interpretation; Drafting Manuscript

\section{(c) (1)(2)}

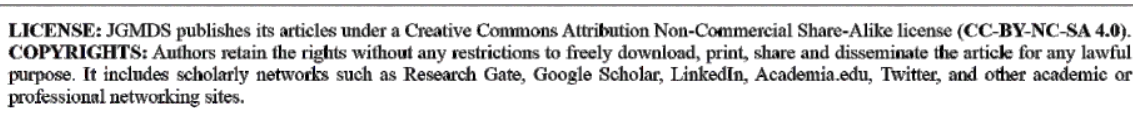

